\title{
Direct measurements of IPTG enable analysis of the induction behavior of $E$. coli in high cell density cultures
}

\author{
Alfred Fernández-Castané, Glòria Caminal and Josep López-Santín
}

\begin{abstract}
Background: The E. coli lac operon and its components have been studied for decades, and lac-derived systems are widely used for recombinant protein production. However, lac operon dynamics and induction behavior remain the paradigm of gene regulation. Recently, an HPLC-MS-based method to quantify IPTG in the medium and inside the biomass has been established, and this tool may be useful to uncover the lack of knowledge and allow optimization of biotechnological processes.

Results: The results obtained from the study of IPTG distribution profiles in fed-batch, high cell density cultures allowed discrimination between two different depletion patterns of an inducer from the medium to the biomass in E. coli-expressing rhamnulose-1-phosphate aldolase (RhuA). Moreover, we could demonstrate that active transport mediates the uptake of this gratuitous inducer. Additionally, we could study the induction behaviors of this expression system by taking into account the biomass concentration at the induction time.

Conclusions: In the bistable range, partial induction occurred, which led to intermediate levels of RhuA activity. There was a direct relationship between the initial inducer concentrations and the initial inducer transport rate together with the specific activity. A majority of the inducer remains in the medium to reach equilibrium with the intracellular level. The intracellular inducer accumulation was a further evidence of bistability of the lac operon.
\end{abstract}

Keywords: IPTG transport, Recombinant protein production, Fed-batch, Bistability, Permease

\section{Background}

Although many experimental studies have been carried out for over 50 years and several mathematical models have been proposed for describing the lac operon regulation, the lac operon remains the paradigm of gene regulation [1-3]. Currently, lac operon regulation is a research objective in systems biology, and lac operon elements are used as tools in biotechnology and synthetic biology [4-7]. For this reason, a detailed knowledge about the lac operon induction behavior is required.

However, no methods have been available to quantify IPTG (Isopropyl- $\beta$-D-1-thiolgalactopyranoside) in the medium or inside the biomass, and assumptions have been made using just the initial concentration of the inducer added to the culture and using reporter genes or

\footnotetext{
*Correspondence: josep.lopez@uab.cat

Departament d'Enginyeria Química, Unitat de Biocatàlisi Aplicada associada al IQAC (CSIC), Universitat Autònoma de Barcelona, Bellaterra, Spain
}

$\beta$-galactosidase activity to give quantitative approaches on lac operon dynamics.

Early studies of the lac operon, which were based on the pioneering work of Cohn \& Horibata [8,9], were concerned with the kinetics of enzyme induction in the presence of gratuitous inducers, such as TMG (thiomethyl galactosides) and IPTG, which cannot be hydrolyzed. These early studies showed that the enzyme synthesis pattern was bistable, with pre-induced cells remaining induced and non-induced cells remaining non-induced $[8,9]$. Furthermore, this bistability disappeared in cryptic mutants lacking lac-permease (lactose permease) [10]. It was proposed that the bistability occurred because of the destabilizing effect of the positive feedback generated by lac-permease that may catalyze the accumulation of the inducer, which in turn stimulates the synthesis of even more permease. Conversely, lactose, the natural inducer of the lac operon, stimulates not only the synthesis of the lac operon enzymes but also cell growth because it is

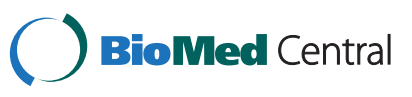


used as a carbon source [11-13]. Recently, Ozbudak et al. [14] performed detailed studies of lac bistability, and they observed that cells exhibited bistability when grown in the presence of succinate and various concentrations of TMG. Moreover, the bistability persisted even if glucose was added to medium containing succinate and TMG, but the thresholds increased with the concentration of extracellular glucose. Laurent et al. [15] developed a mathematical model to explain the bistability of the lac operon and concluded that IPTG may be transported by lac-permease, and therefore, both the medium and intracellular inducer concentrations should be considered. Other recent works stated that IPTG could enter by lac-permease but that a lac-permease-independent entry was possible [16].

However, to understand the induction kinetics, the molecular basis of the lac operon and IPTG induction must be considered [17-19]. In a wild type strain, the lac operon contains two auxiliary operators, $\mathrm{O} 2$ and $\mathrm{O} 3$ (in addition to the main operator), and the repressor is a tetramer containing four inducer-binding sites [19]. Furthermore, repression can also be caused by the formation of DNA loops rather than the repressor-operator binding $[20,21]$. In addition, the bioreactor mixing efficiency may affect the cell population behavior [22].

Noel et al. [23] conducted simulations of kinetic models using the experimental data from Ozbudak and Laurent to study the inducer transport phenomena. It was proposed that carrier efflux could not be ignored for induced cells and diffusive influx could not be neglected for non-induced cells in experiments measuring $\beta$-galactosidase activity [23], and the idea that lac-permease catalyzes IPTG transport is still controversial.

Nevertheless, few studies in the literature take into account the effect of the biomass concentration in the culture when inducing the lac operon, and none account for high cell density cultures. This fact may be of paramount importance when producing heterologous proteins in high cell density cultures. Moreover, no direct measurement of IPTG has been provided to support the hypotheses of the previous studies.

We have recently established an HPLC-MS-based method to measure medium and intracellular IPTG in fedbatch culture samples under substrate-limiting conditions [24]. Moreover, we have demonstrated that lac-permease mediates IPTG transport, and when this transporter is lacking, diffusion is the responsible transport mechanism [25].

In this work, we provide quantitative data of medium and intracellular IPTG concentration evolution in high cell density fed-batch samples in an expression system derived from the lac operon. Distribution profiles of IPTG in the medium as well as the intracellular accumulation of the inducer were analyzed and discussed to provide a better understanding of lac-derived operon dynamics and transport phenomena that are likely involved in the induction of rhamnulose-1-phosphate aldolase (RhuA) expression at different levels of IPTG. Furthermore, we aimed to correlate the induction behavior with the maximum RhuA activity achieved in each experiment, in which we considered that the medium and intracellular inducer concentrations reached equilibrium ("pseudo-steady" state). Our methodology does not provide direct evidence of the bistability of the lac operon but intends to explain the induction patterns of the recombinant protein RhuA in high cell density fed-batch cultures and the possible elements involved.

\section{Results and discussion}

\section{The effect of the biomass concentration}

As stated in the background section, previous studies carried out on the dynamic behavior of the lac operon do not provide experimental data for IPTG and do not report the biomass concentration upon induction in high cell density cultures. This fact should be considered a key parameter because inducer uptake may be affected by the number of cells capable of incorporating the inducer within the cytoplasm. These early experiments were performed on a shake flask scale with a low concentration of biomass. In contrast, this work addresses an auxotrophic expression system for production of RhuA in fed-batch cultures using a predefined exponential feeding profile to reach biomass concentrations at production scales. Moreover, one has to take into account that this system harbors two plasmids (pQE $\alpha \beta$ rham and pREP4) expressing the LacI protein constitutively and has more operator sites than the wild type strain. Plasmid copy number of $\mathrm{pQE} \alpha \beta$ rham and pREP4 is $30-40$ and 10-12, respectively [26].

Therefore, it was convenient to assess the influence of cell concentration on IPTG uptake. Table 1 summarizes the results obtained for RhuA production in terms of specific activity and initial transport rate values $\left(\mathrm{q}_{\mathrm{I}}\right)$ for two different biomass concentrations and inducing RhuA with IPTG at 20 and $100 \mu \mathrm{M}$. Using the same inducer concentration, lower specific activities were obtained upon induction at $40 \mathrm{~g}$ DCW $\cdot \mathrm{L}^{-1}$ than when the biomass concentration at induction was $20 \mathrm{~g} \mathrm{DCW} \cdot \mathrm{L}^{-1}$. Similar results were obtained for the two inducer levels studied. Table 1 also shows a clear dependency of the initial uptake rate of IPTG from medium

\begin{tabular}{|c|c|c|c|c|}
\hline Ferm.code & $\begin{array}{l}{[\mathrm{IPTG}]_{0}} \\
(\mu \mathrm{M})\end{array}$ & $\begin{array}{c}X_{\text {ind }} \\
\left(g \text { DCW. } L^{-1}\right) \\
\end{array}$ & $\begin{array}{l}\text { RhuA activ. } \\
\left(\mathrm{AU} \cdot \mathrm{g}^{-1} \mathrm{DCW}\right)\end{array}$ & $\begin{array}{c}q_{10} \\
\left(\mu M \cdot h^{-1}\right)\end{array}$ \\
\hline FB3 & 20 & 20 & 769 & 5 \\
\hline FB3B & 20 & 40 & 380 & 1478 \\
\hline FB6 & 100 & 20 & 1028 & 350 \\
\hline FB6B & 100 & 40 & 513 & 2141 \\
\hline
\end{tabular}


on the biomass concentration at induction. The higher biomass leads to a faster entry of IPTG into the cell. Therefore, the biomass concentration of a culture should be considered when studying lac operon dynamics in cell populations. Consequently, the degree of induction not only depends on the environmental conditions, i.e., IPTG concentration in the medium but also on the concentration of the biomass population that may compromise the inducer availability for a single cell at low concentrations of the inducer.

\section{Distribution profiles of IPTG in medium and inside the biomass: induction behavior}

A study of the induction behavior using fed-batch experiments was performed under the same conditions, with the only variation being the initial inducer concentration $(8-1000 \mu \mathrm{M})$. We could observe two different patterns of IPTG depletion from the medium upon induction (see Additional file 1). In fermentations induced with up to $40 \mu \mathrm{M}$, IPTG decreased from the medium gradually, resulting in initial transport rate $\left(\mathrm{q}_{\mathrm{IO}}\right)$ values of 3-5 $\mu \mathrm{M} \cdot \mathrm{h}^{-1}$; however, upon induction at higher concentrations, IPTG is initially depleted from the medium rapidly, resulting in initial transport rates of 300 to $1400 \mu \mathrm{M} \cdot \mathrm{h}^{-1}$. q Io values were calculated as the initial slope of the extracellular IPTG profiles (see Additional file 1), which are shown in Figure 1. The number of sonication cycles for disruption of biomass samples and subsequent release of intracellular inducer was assessed. After four sonication cycles maximum IPTG levels were measured. The increase of the number of sonication cycles within a sample did not lead to a further release of the inducer. Distribution profiles of intracellular IPTG at different initial inducer concentration levels at induction time were evaluated. The intracellular inducer concentration increases up to a certain concentration, although, in certain cases, the intracellular IPTG first increases and then decreases.

The two differential patterns of inducer depletion from the medium can be attributed to the different mechanisms of transport involved in IPTG uptake. The cases in which intracellular IPTG increases to a certain concentration and then decreases could be due to the feedstock supply to the reactor and to the increase of cell volume with cellular division. Additionally, cell lysis could promote the release of the inducer into the extracellular medium. The employed analytical method for IPTG can only measure free IPTG, and not the one bound to other molecules.

A comparison between the initial transport rate and specific activity values at the pseudo-steady state was performed. The results shown in Figure 1 indicate that low qio values were obtained at initial inducer concentrations corresponding to 8 to $40 \mu \mathrm{M}$ IPTG and that coincided with intermediate levels of induction. The maximal activity of recombinant protein was obtained above $40 \mu \mathrm{M}$ IPTG, which coincided with higher $\mathrm{q}_{\mathrm{Io}}$ values (Figure 2). Therefore, we suggest that the full induction of RhuA is achieved above 20 to $40 \mu \mathrm{M}$ IPTG. According to previously published results [25], we postulate that lac-permease has a lesser role at low initial inducer concentrations (partial induction) than at relatively high values of initial inducer concentration. Our results are in agreement with the theoretical model of the dynamic behavior of the lac operon that was proposed by Laurent [15]. It is likely that above a threshold value, which was $40 \mu \mathrm{M}$ IPTG in our system, the transcription of permease is completely triggered, consequently enhancing the uptake of the inducer, and subsequently, RhuA activity reaches its maximum values. Nevertheless, active transport may occur at all of the inducer concentrations studied because the measured intracellular concentrations of inducer $\mathrm{IPTG}_{0}(8-10 \mu \mathrm{M})$ were higher than in the medium at the "pseudo-steady" state, as shown in Figure 3.

\section{Comparison of the medium and intracellular IPTG concentrations in the "pseudo-steady" state}

The concentrations of the inducer in the medium and intracellular are shown in Figure 3, when RhuA was at a maximum. As anticipated, upon induction at 8-10 $\mu \mathrm{M}$ IPTG, the intracellular concentration of the inducer was higher than that in the medium, but reached similar values at $20 \mu \mathrm{M}$ IPTG. At higher initial concentrations of IPTG, the medium values were always higher than those inside the biomass.

When there was no inducer, $[\mathrm{IPTG}]_{\text {intra }}=[\mathrm{IPTG}]_{\text {extra }}=0$, a steady state (SS1). In our expression system, we can assume that at low concentrations of IPTG, the initial inducer concentration is so low that it cannot enter the cell $\left([\mathrm{IPTG}]_{\text {intra }}<[\mathrm{IPTG}]_{\text {extra }}\right)$. From previous experiments performed by our research group, we know that there is

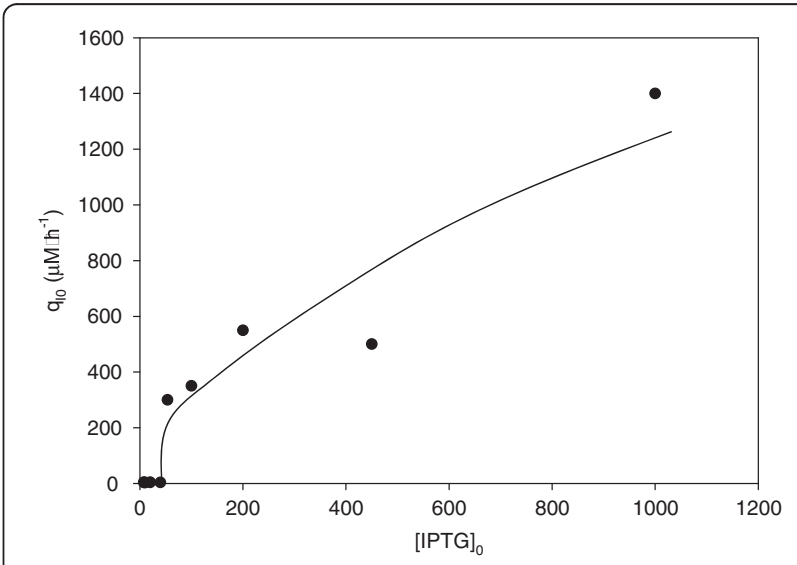

Figure 1 Initial IPTG transport rate $\left(q_{10}, \mu \mathrm{M} \cdot \mathrm{h}^{-1}\right)$ from medium to the biomass vs. initial inducer concentration (IPTG $\left.{ }_{0}, \mu \mathrm{M}\right)$ in fed-batch substrate limiting experiments. Each point corresponds to the initial slope of IPTG medium concentration profile. 


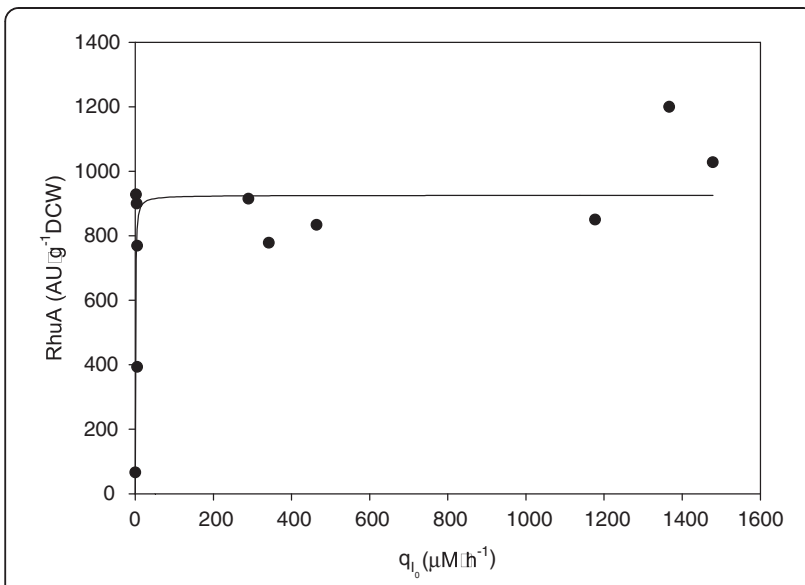

Figure 2 Dependency of specific RhuA activity $\left(\mathrm{AU} \cdot \mathrm{g}^{-1} \mathrm{DCW}\right)$ at "pseudo-steady" state on the initial inducer transport rate ( $q_{10}$ $\mu \mathrm{M} \cdot \mathrm{h}^{-1}$ ) from medium to the biomass.

no RhuA overexpression upon induction at IPTG concentrations lower than $4 \mu \mathrm{M}$ [26]. By increasing the extracellular inducer, IPTG can enter the cell by diffusion and/or active transport and can accumulate until it reaches a second steady state (SS2) at concentrations $\leq 4 \mu \mathrm{M}$, being SS2 the "on" threshold to induce RhuA. Then, the intracellular concentration overcomes the medium concentration at 8$10 \mu \mathrm{M}\left([\mathrm{IPTG}]_{\text {intra }}>[\mathrm{IPTG}]_{\text {extra }}\right)$. At this stage, RhuA expression is not fully induced because the intracellular inducer does not bind the repressor with an affinity strong enough to release all of the repressor molecules from all of the operator sites. Another possible explanation is that there are some cells induced and others remain noninduced when using low concentrations of IPTG. By

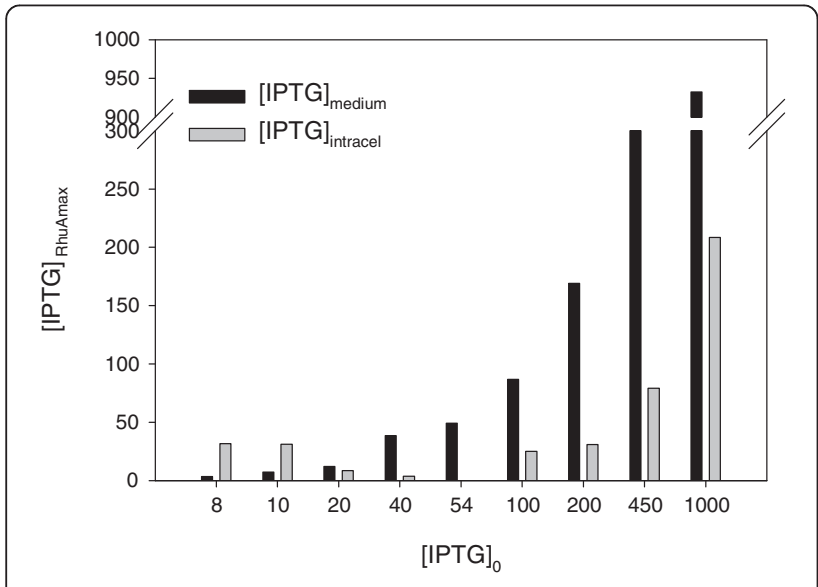

Figure 3 Comparison of medium and intracellular IPTG concentrations $(\mu \mathrm{M})$ when specific RhuA $\left(A U \cdot g^{-1} D C W\right)$ is maximum ("pseudo-steady" state) at different initial inducer concentrations (IPTG ${ }_{0}, \boldsymbol{\mu M}$ ). The analytical standard error for experimental IPTG measurements is 5 and $7 \%$ for medium and intracellular samples, respectively. increasing the initial inducer concentration, the free intracellular inducer concentration decreases because it binds to the repressor (cannot be measured) and because of cell division. Notably, the repressor-inducer binding cannot be measured by the HPLC-MS analytical method. Subsequently, the equilibrium between the medium and intracellular concentrations is reached upon induction at $20 \mu \mathrm{M}$ (SS3), where partial induction still occurs. Above this concentration, full induction is achieved because all of the repressor is released from the operator sites. Therefore, for initial inducer concentration $>40 \mu \mathrm{M},[\text { IPTG }]_{\text {intra }}<[\text { IPTG }]_{\text {extra }}$. Our results are compatible with the assumptions concerning the IPTG concentrations in the medium and inside the biomass by Laurent and co-workers [15] and are in agreement with other theoretical hypotheses $[3,14,27,28]$.

Figure 3 also shows a direct dependency of the measured concentration of the inducer in the medium when RhuA is at a maximum ("pseudo-steady" state) with the initial IPTG added to the reactor. However, the measured intracellular IPTG has no direct dependency on the initial inducer concentration in the "pseudo-steady" state. This fact could be due to the dynamics of the lac operon, as explained previously. The free intracellular inducer accumulates, and when a certain concentration is reached, intracellular IPTG can bind all of the repressor. This fact explains the intracellular depletion of the inducer. After all of the repressor is bound to the inducer, the free intracellular inducer concentration increases and accumulates when the full induction of RhuA is achieved.

By measuring the concentration of IPTG in the medium, we also demonstrate that at any initial concentration of the inducer added to the culture, there is an excess of IPTG because the majority of it remains in the medium at "pseudo-steady" state, as shown in Figure 4. It is seen that, except upon induction at $8 \mu \mathrm{M}$ IPTG, the majority of the inducer remains in the medium and that at high initial concentrations, only a minor amount of the inducer is taken up by the biomass.

Transitions between the non-induced and induced states were interpreted by Cohn \& Horibata in their experiments with the lac operon. From their results, theoretical studies showed that hysteresis is an inherent property of bistable systems. However, it has been shown that $E$. coli populations were heterogeneous in terms of lac induction around the transition $[8,16,29]$. For our expression system, the bistable range (8-40 $\mu \mathrm{M}$ IPTG), where only partial induction is achieved, can be attributed to two main reasons. First, not all of the repressor is removed from the operator site in induced cells. Second, it is likely that not all of the cell population is induced due to the low availability of external IPTG. Whatever the cause, it leads to intermediate levels of RhuA activity, as shown previously in Figure 2. Additionally, from the production point of view, the 


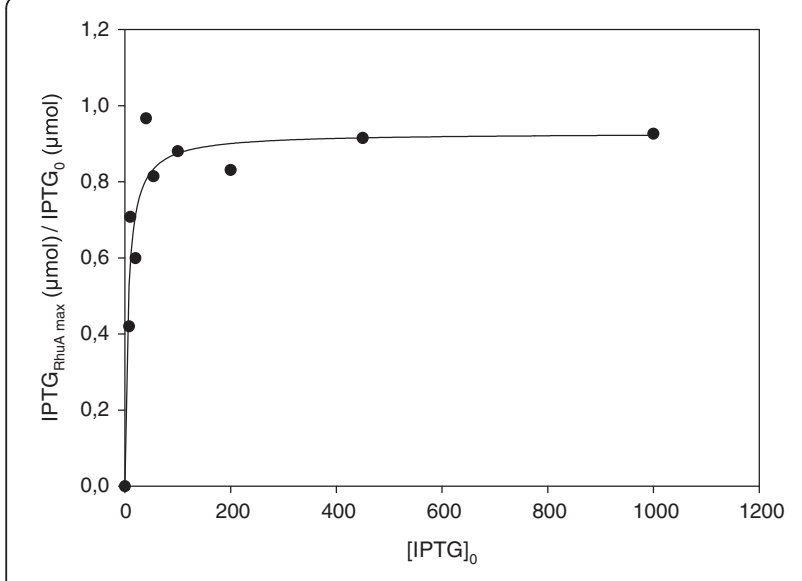

Figure 4 Fraction of IPTG remaining in medium when specific RhuA activity $\left(A U \cdot \mathrm{g}^{-1} \mathrm{DCW}\right)$ is maximum vs. the initial IPTG concentration (IPTG,$\mu \mathrm{M})$ added into the reactor. concentration added to the reactor (Figure 5B). The behavior presented in Figure 5 could be further evidence of bistability patterns. For the non-fully induced range (IPTG $\leq 40 \mu \mathrm{M}$ ), induced and non-induced cells were expected to be present after induction. Alternatively, in this range, cell growth was not as affected by the RhuA overexpression as by the full induction. Cell growth decreases are dependent upon the initial inducer concentration for our expression system [30]. The experimental observations in this range lead to a pattern compatible with the model proposed by Noel et al. [23], which considered the effect of active transport and diffusion of the inducer. However, their simulations were based on experimental data for TMG that were obtained from Ozbudak et al. [14], in which arbitrary units for the inducer and permease activity in logarithmic scales were given. Our experimental results show great parallelisms with the simulations proposed in these studies.

\section{Missing inducer and estimation of inducer-repressor affinity}

As indicated before, the analytical method used to quantify IPTG inside the biomass measured the free intracellular IPTG, and it was not possible to measure the inducer bound to other molecules. Therefore, we have calculated the amount of IPTG missing at each concentration level in terms of total $\mu \mathrm{mol} \cdot \mathrm{g}^{-1} \mathrm{DCW}$ (data not shown). There is a lack of $0-1.2 \mu \mathrm{mol} \cdot \mathrm{g}^{-1} \mathrm{DCW}$, which is dependent on the experiment, that represents $0-30 \%$ of the total inducer added. However, it is known that certain amounts of IPTG are bound to the repressor protein, to the permease or to other unspecific proteins. A first approach could be to
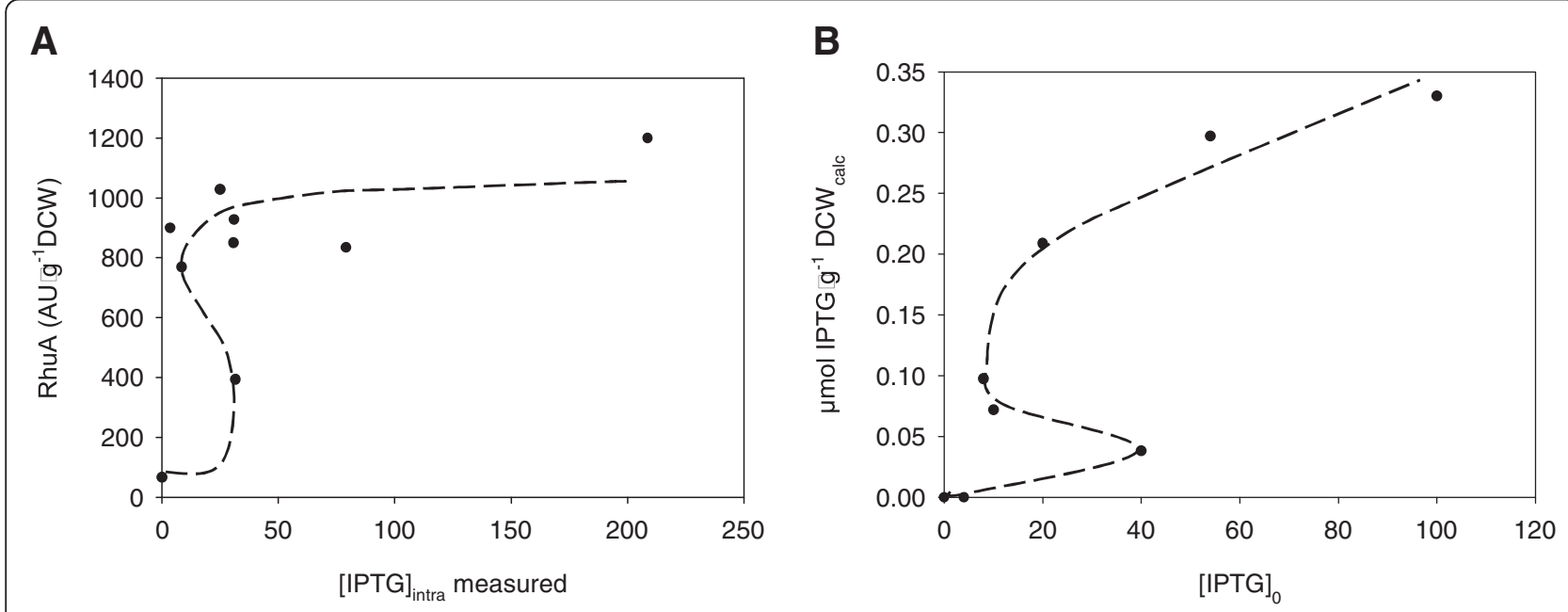

Figure 5 Comparison of the "pseudo-steady" states. A) Variation of the maximum specific RhuA activities (AU. " $\left.^{-1} D C W\right)$ with the measured intracellular IPTG concentration ( $\mu$ M). B) Variation of the calculated intracellular inducer ( $\mu$ mol IPTG. $g^{-1} D C W$ ) with the initial IPTG concentration $\left(\mathrm{IPTG}_{0}, \mu \mathrm{M}\right)$ added into the reactor. The analytical standard error for experimental IPTG measurements is 5 and $7 \%$ for medium and intracellular samples, respectively. 
assume that the inducer amount that was not measured was due to the IPTG-repressor binding. Therefore, we have estimated the inducer-repressor affinity inside the cell.

The equilibrium between free and bound inducer can be postulated as follows:

$$
\begin{array}{r}
\text { IPTG }+\mathrm{R} \rightleftarrows \mathrm{IPTG}-\mathrm{R} \\
K c=\frac{[I P T G-R]}{[I P T G][R]}
\end{array}
$$

where

[IPTG]= free intracellular IPTG concentration

$[R]=$ repressor concentration

[IPTG-R] = inducer-repressor concentration

and, conversely, the total repressor concentration $\left([R]_{\text {Total }}\right)$ can be expressed as the addition of free and bound ones as follows:

$$
[R]+[I P T G-R]=[R]_{\text {Total }}
$$

By substituting $[R]$ from Equation 1 in to Equation 2 and assuming that [IPTG-R] can be estimated as the amount of missing intracellular inducer, as follows:

$$
\frac{[I P T G-R]}{[I P T G]}=\frac{I P T G_{\text {intracel }} \text { missing }}{I P T G_{\text {intracel }} \text { measured }}
$$

By utilizing the data from all experiments, a linear relationship should be obtained that allows the determination of $K_{c}$ and $[R]_{\text {Total }}$. As is seen in Figure 6, the fit was not good. Nevertheless, with the necessary cautions, "rough" values of $\mathrm{K}_{\mathrm{c}}$ and $[R]_{\text {Total }}$ can be estimated. The inaccurate fitting could be due to the assumption that all of the missing intracellular inducer is bound to the repressor. Furthermore,

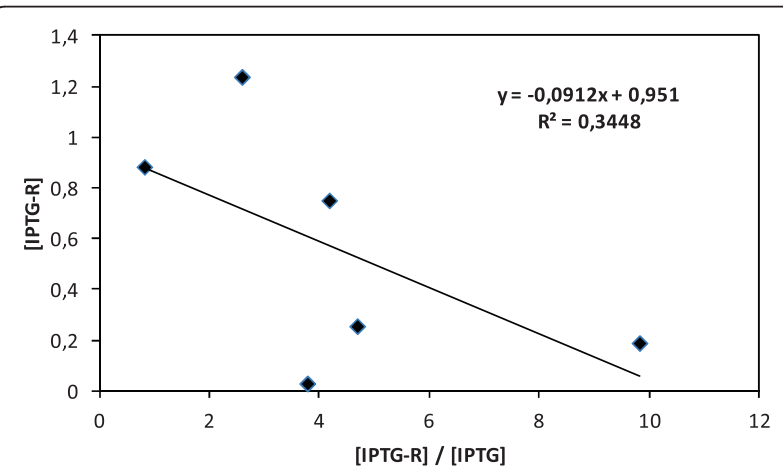

Figure 6 Bound inducer concentration (calculated as inducer missing concentration) vs. the ratio between bound inducer concentration/measured inducer concentrations. The slope corresponds to $-1 / k_{c}$. The intercept corresponds to total repressor concentration. The analytical standard error for experimental IPTG measurements is 5 and $7 \%$ for medium and intracellular samples, respectively. it is likely that experimental error and the amount of IPTG bound to other molecules could affect the obtained result.

The dissociation constant for the repressor-inducer binding is $\mathrm{K}_{\mathrm{c}}^{-1} \approx 4300 \mu \mathrm{M}$. The approximate estimation from an experimental measurement of IPTG was quite different from the ones proposed in the literature, which are $30 \mu \mathrm{M}$ [21] or $7-30 \mu \mathrm{M}$ [31]. The approximate calculated concentration of the total repressor was $430 \mu \mathrm{M}$, in contrast with the $0.02 \mu \mathrm{M}$ calculated by Dunaway and co-workers. This significant difference in the order of magnitude is compatible with the higher number of LacI copies that were encoded in our expression system (pREP4 plasmid) and the higher number of repressor-operator sites encountered in the $\mathrm{pQE} \alpha \beta$ rham plasmid in contrast with the strains studied by the other authors. From our results, we suggest a 10:1 inducer-repressor stoichiometry of induction, which is much higher than that suggested by Oehler and co-workers, indicating that our expression system is tightly regulated. However, the $\mathrm{K}_{\mathrm{c}}$ and total repressor values obtained in this work were not only approximated but also overestimated because the inducer is likely bound to other molecules. It is worth noting that the order of magnitude is compatible with the calculated intracellular inducer concentration for the full induction range (see Additional file 1). Alternatively, a more rigorous treatment of this approximation would require taking into account the operatorrepressor and inducer-repressor-operator bindings $[17,18]$. Additionally, as stated previously, our results are not comparable with those from the literature because we are dealing with a complex expression system to produce RhuA differently than wild type strains.

\section{Conclusions}

For the first time, the induction behavior of a lac-derived operon was assessed using experimental data for IPTG at biomass concentrations employed to produce recombinant proteins. In the bistable range, in which a partial induction occurs, intermediate levels of RhuA activity were achieved. Full induction was achieved at IPTG concentrations greater than $40 \mu \mathrm{M}$. There was a direct relationship between the initial inducer concentrations and the initial inducer transport rate together with the specific activity. A majority of the inducer remains in the medium to reach equilibrium with the intracellular level. The intracellular inducer accumulation could be a further evidence of bistability of the lac operon and its inherent hysteresis. A comparison of our results with data from the literature was not a trivial task because there was no method available for quantifying IPTG and we utilized an expression system for recombinant protein production at high cell density cultures. Therefore, extensive studies of the inducer transport under different conditions (i.e., operation conditions or biomass concentrations) or using different 
strains could be of interest. To summarize, this study on IPTG transport provides experimental data for developing mathematical models that are able to predict the distribution profiles of an inducer together with the production of recombinant proteins that are of industrial interest.

\section{Methods}

Media, chemicals and other reagents

LB (Lennox Broth) medium, which is composed of $10 \mathrm{~g} \mathrm{~L}^{-1}$ peptone, $5 \mathrm{~g} \mathrm{~L}^{-1}$ yeast extract and $10 \mathrm{~g} \mathrm{~L}^{-1} \mathrm{NaCl}$, was used for the pre-cultures. A stock solution of ampicillin (100 $\mathrm{mg} \mathrm{mL}^{-1}$; Sigma) was prepared in 50\% (v/v) ethanol, filter sterilized through a Millex ${ }^{\circledR} \mathrm{GS} \quad 0.22-\mu \mathrm{m}$ filter from Millipore and stored at $-20^{\circ} \mathrm{C}$. Isopropyl $\beta-\mathrm{D}$ 1-thiogalactoryranoside was purchased from SigmaAldrich, and a stock solution was prepared by dissolving $2.38 \mathrm{~g}$ in $100 \mathrm{~mL}$ of Milli-Q water that was filter sterilized through a Millex ${ }^{\circledR}$ GS $0.22-\mu$ m filter from Millipore to obtain a stock solution of $100 \mathrm{mM}$ and stored at $-20^{\circ} \mathrm{C}$. The composition of the Defined Medium (DM) for the shake flask cultures and fed-batch fermentations were described elsewhere [32]. Formic acid (98\% purity) was supplied from Panreac, and Milli-Q water was employed for the HPLC analysis.

\section{Strain, fermentation and growth conditions}

The E. coli M15 $\Delta g l y A$ [pREP4] pQE $\alpha \beta$ rham expression system was used as the parental strain for RhuA production. Construction of the pQE $\alpha \beta$ rham vector for the production of RhuA under the control of the strong promoter T5 was described elsewhere [33]. This expression system is based on glycine auxotrophy to ensure plasmid stability and avoid antibiotic supplementation. A detailed explanation of the fed-batch substrate-limiting cultures can be found elsewhere [32,34]. A defined medium with glucose as the sole carbon source $\left(20 \mathrm{~g} \cdot \mathrm{L}^{-1}\right)$ was used for the batch phase. Once the substrate was consumed, a fed-batch phase starts supplying a feedstock solution to maintain a constant and specific growth rate of $0.22 \mathrm{~h}^{-1}$ by using a predefined exponential feeding profile. The overexpression of RhuA was pulse induced at 20 or $40 \mathrm{~g} \mathrm{DCW} \cdot \mathrm{L}^{-1}$ with different initial IPTG concentrations. Table 2 shows the eleven experiments that were performed under the same conditions, with only the initial inducer concentration and biomass concentration at induction time varying.

The volume of the cells was calculated according to the biomass concentration and by assuming a volume of $0.0023 \mathrm{~L} \mathrm{~g}^{-1} \mathrm{DCW}$ [35] to enable the calculation of IPTG concentration inside the biomass.

\section{Analytical methods}

The E. coli growth was monitored by optical density measurements at a wavelength of $600 \mathrm{~nm}$ using a
Table 2 The fed-batch fermentations performed at different inducer concentration levels and the biomass concentration at induction time

\begin{tabular}{|c|c|}
\hline Fermentation code & 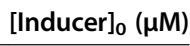 \\
\hline $\mathrm{FB} 1^{\mathrm{a}}$ & 8 \\
\hline $\mathrm{FB} 2^{\mathrm{a}}$ & 10 \\
\hline $\mathrm{FB} 3^{\mathrm{a}}$ & 20 \\
\hline $\mathrm{FB} 4{ }^{\mathrm{a}}$ & 40 \\
\hline $\mathrm{FB} 5^{\mathrm{a}}$ & 54 \\
\hline $\mathrm{FB6}^{\mathrm{a}}$ & 100 \\
\hline $\mathrm{FB} 7^{\mathrm{a}}$ & 200 \\
\hline $\mathrm{FB} 8^{\mathrm{a}}$ & 450 \\
\hline FB9 ${ }^{a}$ & 1000 \\
\hline $\mathrm{F} 3 \mathrm{~B}^{\mathrm{b}}$ & 20 \\
\hline $\mathrm{F} 6 \mathrm{~B}^{\mathrm{b}}$ & 100 \\
\hline
\end{tabular}

a Experiments induced at $20 \mathrm{~g} \mathrm{DCW} \cdot \mathrm{L}^{-1}$

${ }^{b}$ Experiments induced at $40 \mathrm{~g} \mathrm{DCW} \cdot \mathrm{L}^{-1}$.

spectrophotometer (Uvicon 941 Plus, Kontrol). Samples were diluted with distilled water until the final $\mathrm{OD}_{600 \mathrm{~nm}}$ value was within the range 0.3-0.9. The biomass was expressed as dry cell weight (DCW), with $1 \mathrm{OD}_{600 \mathrm{~nm}}$ being equivalent to $0.3 \mathrm{~g} \cdot \mathrm{L}^{-1} \mathrm{DCW}$ [36]. The cell disruption and quantitation of RhuA activity protocols are described elsewhere [37]. The IPTG analysis was performed on a Shimadzu Prominence liquid chromatograph with a UV/Vis detector operating at a wavelength of $210 \mathrm{~nm}$, which was coupled to a mass spectrometer Shimadzu 2010A equipped with an ESI (electrospray ionization) interface and single quadrupole, and using a LC-10 AD solvent delivery system (pump A and B). A Shimadzu FCV-20 H2 valve unit was used to divert the flux. The injection was made with a Shimadzu SIL$10 \mathrm{AD}$ automatic injector, and the data analysis was processed with Lab Solutions 3.04 software. Samples were kept in the autoinjector at room temperature. The sample preparation and chromatographic and mass spectrometer conditions are described elsewhere [24].

\section{Determination of IPTG concentration in fermentation samples}

For medium samples, the concentration of the inducer measured by HPLC-MS was multiplied by the dilution factor. To obtain the total $\mu \mathrm{mol}$ of IPTG in the medium, the concentration was multiplied by the volume of medium inside the reactor, which can be determined by the difference between the measured total volume and the volume of the cells. The volume of the cells was calculated from the biomass concentration and by employing a specific volume of $0.0023 \mathrm{~L} \cdot \mathrm{g}^{-1} \mathrm{DCW}$ [35]. The biomass concentration was calculated by measuring $\mathrm{OD}_{600 \mathrm{~nm}}$ and knowing that $1 \mathrm{OD}_{600 \mathrm{~nm}}$ has an equivalence of $0.3 \mathrm{~g} \mathrm{DCW} \cdot \mathrm{L}^{-1}[36]$. 
The intracellular samples were adjusted to an $\mathrm{OD}_{600 \mathrm{~nm}}$ of 4 before cell disruption and subsequent IPTG analysis was performed. The intracellular concentration of the inducer could be obtained from the IPTG analysis as the biomass concentration and the equivalence to the DCW were known. The total intracellular IPTG in terms of $\mu \mathrm{mol}$ could also be determined because the biomass concentration was known and the cell volume could be assumed.

For the range of IPTG concentrations studied, the associated standard error was 5 and $7 \%$ for the medium and intracellular samples, respectively. The development and validation of IPTG analysis in medium and intracellular fed-batch fermentations was previously validated under the FDA (Food and Drug Administration) guidelines [24].

\section{Additional file}

Additional file 1: Distribution profiles of IPTG in the medium and intracellular fed-batch samples together with biomass evolution and RhuA production of the nine experiments performed under different initial inducer concentration. Time zero corresponds to the induction time.

\section{Competing interests}

The authors declare that they do not have any competing interests.

\section{Acknowledgements}

This work has been supported by the Spanish MICINN, projects CTQ2008-00578 and CTQ2011-28398-C02-01, and by DURSI 2009SGR281 Generalitat de Catalunya.

The Department of Chemical Engineering of UAB constitutes the Biochemical Engineering Unit of the Reference Network in Biotechnology of the Generalitat de Catalunya (XRB).

AFC acknowledges UAB for a pre-doctoral grant.

\section{Authors' contributions}

AFC contributed to all of the experiments and manuscript preparation. GC and JLS participated in the research design and manuscript preparation. All of the authors have read and approved the final manuscript.

Received: 2 March 2012 Accepted: 3 April 2012

Published: 9 May 2012

\section{References}

1. Beckwith J: Escherichia coli and Salmonella typhimurium. In The lactose operon, Volume 2. Edited by Neidhardt FC American Society for Microbiology. 1987:1444-1452.

2. Lee SY: High cell-density culture of Escherichia coli. Trends Biotechnol 1996, 14:98-105.

3. Vilar JMG, Guet CC, Leibler S: Modeling network dynamics: the lac operon, a case study. J Cell Biol 2003, 161:471-476.

4. Shiloach J, Kaufman J, Guillard a S, Fass R: Effect of glucose supply strategy on acetate accumulation, growth, and recombinant protein production by Escherichia coli BL21 (lambdaDE3) and Escherichia coli JM109. Biotechnol Bioeng 1996, 49:421-428.

5. Sandén AM, Boström M, Markland K, Larsson G: Solubility and proteolysis of the Zb-MalE and Zb-MalE31 proteins during overproduction in Escherichia coli. Biotechnol Bioeng 2005, 90:239-247.

6. Ishikawa M, Koizumi S: Microbial production of N-acetylneuraminic acid by genetically engineered Escherichia coli. Carbohyd Res 2010, 345:2605-2609.

7. Volontè F, Piubelli L, Pollegioni L: Optimizing HIV-1 protease production in Escherichia coli as fusion protein. Microb Cell Fact 2011, 10:53.

8. Novick A, Weiner M: Enzyme induction as an all-or-none phenomenon. Proc Natl Acad Sci U S A 1957, 43:553-566.
9. Cohn M, Horibata K: Inhibition by glucose of the induced synthesis of the beta-galactoside-enzyme system of Escherichia coli. Analysis of maintenance. J Bacteriol 1959, 78:601-612.

10. Herzenberg L: Studies on the induction of beta-galactosidase in a cryptic strain of Escheriachia coli. Biochim Biophys Acta 1959, 31:525-538.

11. Abeles RH, Frey PA, Jencks WP: Biochemistry. Boston: Jones and Bartlett; 1992:838.

12. Donovan RS, Robinson CW, Glick BR: Review: optimizing inducer and culture conditions for expression of foreign proteins under the control of the lac promoter. J Ind Microbiol 1996, 16:145-154.

13. de Vos WM: Systems solutions by lactic acid bacteria: from paradigms to practice. Microb Cell Fact 2011, 10(Suppl 1):S2.

14. Ozbudak EM, Thattai M, Lim HN, Shraiman BI, Van Oudenaarden A: Multistability in the lactose utilization network of Escherichia coli. Nature 2004, 427:737-740

15. Laurent M, Charvin G, Guespin-Michel J: Bistability and hysteresis in epigenetic regulation of the lactose operon. Since Delbrück, a long series of ignored models. Cell Mol Biol 2005, 51:583.

16. Marbach A, Bettenbrock K: lac operon induction in Escherichia coli: Systematic comparison of IPTG and TMG induction and influence of the transacetylase LacA. J Biotechnol 2012, 157:82-88.

17. Dunaway M, Olson JS, Rosenberg JM, Kallai OB, Dickerson RE, Matthews KS: Kinetic studies of inducer binding to lac repressor.operator complex. J Biol Chem 1980, 255:10115-10119.

18. O'Gorman RB, Rosenberg JM, Kallai OB, Dickerson RE, Itakura K, Riggs a D, Matthews KS: Equilibrium binding of inducer to lac repressor.operator DNA complex. J Biol Chem 1980, 255:10107-10114.

19. Lewis M: The lac repressor. CR Biol 2005, 328:521-548.

20. Kao-Huang Y, Butler a P, O'Conner P, Noble DW, von Hippel PH: Nonspecific DNA binding of genome-regulating proteins as a biological control mechanism: measurement of DNA-bound Escherichia coli lac repressor in vivo. Proc Natl Acad Sci U S A 1977, 74:4228-4232.

21. Oehler $S$, Alberti $S$, Müller-Hill B: Induction of the lac promoter in the absence of DNA loops and the stoichiometry of induction. Nucleic Acids Res 2006, 34:606-612.

22. Delvigne $F$, Boxus M, Ingels S, Thonart P: Bioreactor mixing efficiency modulates the activity of a prpoS::GFP reporter gene in E. coli. Microb Cell Fact 2009, 8:15.

23. Noel JT, Pilyugin SS, Narang A: The diffusive influx and carrier efflux have a strong effect on the bistability of the lac operon in Escherichia coli. J Theor Biol 2009, 256:14-28.

24. Fernández A, Ruiz J, Caminal G, López-Santín J: Development and validation of a liquid chromatography-mass spectrometry assay for the quantitation of IPTG in E. coli fed-batch cultures. Anal Chem 2010, 82:5728-5734

25. Fernández-Castané $A$, Vine CE, Caminal G, López-Santín J: Evidencing the role of lactose permease in IPTG uptake by Escherichia coli in fed-batch high cell density cultures. J Biotechnol 2012, 157:391-398.

26. Pinsach J: Development of recombinant aldolase production process in Escherichia coli. PhD thesis. 2009. 170 pages.

27. Müller J, Barker A, Oehler S, Müller-Hill B: Dimeric lac repressors exhibit phase-dependent co-operativity. J Mol Biol 1998, 284:851-857.

28. Kaback HR, Sahin-Tóth M, Weinglass a B: The kamikaze approach to membrane transport. Nat Rev Mol Cell Biol 2001, 2:610-620.

29. Andersson L, Yang S, Neubauer P: Impact of plasmid presence and induction on cellular responses in fed batch cultures of Escherichia coli. J Biotechnol 1996, 46:255-263.

30. Ruiz J, González G, de Mas C, López-Santín J: A semiempirical model to control the production of a recombinant aldolase in high cell density cultures of Escherichia coli. Biochem Eng J 2011, 55:82-91.

31. Narang A: Effect of DNA looping on the induction kinetics of the lac operon. J Theor Biol 2007, 247:695-712.

32. Ruiz J, Pinsach J, Álvaro G, González G, de Mas C, Resina D, López-Santín J: Alternative production process strategies in E. coli improving protein quality and downstream yields. Process Biochem 2009, 44:1039-1045.

33. Vidal L, López-Santin J, Caminal G, Ferrer P: Development of an antibiotic-free plasmid selection system based on glycine auxotrophy for recombinant protein overproduction in Escherichia coli. [Poster presentation]. Microb Cell Fact 2006, 5:P85.

34. Pinsach J, De Mas C, Lopez-Santin J: A simple feedback control of Escherichia coli growth for recombinant aldolase production in fed-batch mode. Biochem Eng J 2006, 29:235-242. 
35. Vidal L, Ferrer $P$, Alvaro G, Benaiges MD, Caminal G: Influence of induction and operation mode on recombinant rhamnulose 1-phosphate aldolase production by Escherichia coli using the T5 promoter. J Biotechnol 2005, 118:75-87.

36. Vidal L, Pinsach J, Striedner G, Caminal G, Ferrer P: Development of an antibiotic-free plasmid selection system based on glycine auxotrophy for recombinant protein overproduction in Escherichia coli. J Biotechnol 2008, 134:127-136.

37. Bennett BD, Yuan J, Kimball EH, Rabinowitz JD: Absolute quantitation of intracellular metabolite concentrations by an isotope ratio-based approach. Nat Protoc 2008, 3:1299-1311.

\section{doi:10.1186/1475-2859-11-58}

Cite this article as: Fernández-Castané et al:: Direct measurements of IPTG enable analysis of the induction behavior of $E$. coli in high cell density cultures. Microbial Cell Factories 2012 11:58.

\section{Submit your next manuscript to BioMed Central and take full advantage of:}

- Convenient online submission

- Thorough peer review

- No space constraints or color figure charges

- Immediate publication on acceptance

- Inclusion in PubMed, CAS, Scopus and Google Scholar

- Research which is freely available for redistribution 